\title{
Privacy-Preserving Auditing Protocol for Dynamic Group Cloud Environment
}

\author{
Mahesh Ashok Shinde ${ }^{1}$, Y. B. Gurav ${ }^{2}$ \\ ${ }^{1}$ PG Student, Computer Department, Padmabhooshan Vasantdada Patil Institute of Technology, Bavdhan, Pune, India \\ ${ }^{2}$ Assistant Professor, Computer Department, Padmabhooshan Vasantdada Patil Institute of Technology, Bavdhan, Pune, India
}

\begin{abstract}
By cause of the hardware/software falsers and mortal flaws, with cloud information services, information isn't solely keep in cloud, however additionally multiple users will shared across; the virtue of cloud knowledge is prone to scepticism. Many mechanisms are drafted to consent information homeowners and public verifiers to earnestly accomplish intact checking while not downloading the complete knowledge in distinction to the cloud server that is noted as a public auditing. But together with his existing mechanisms this crowed reviewing on the intact of communal knowledge together, to crowed provers can inevitably reveal wind like identity privacy. For crowed reviewing on communal knowledge keep inside the cloud, a replacement offbeat secrecy-sustaining contivance is helpfull. It is needed to review the correctness of communal knowledge for this coup ring mark to reckon verification information. With this mechanism, on exclusive area in communal knowledge, the coherance of the endorser is unbroken personal from crowed provers, whereas not reclaiming the whole file, who square measure ready to efficiently verify shared data integrity. In addition to the existing mechanism is during a position instead of supportive them one by one to perform different reviewing scripts at identical time.
\end{abstract}

Keywords: Cloud Storage, Privacy Preserving, Third party auditor, Secret key

\section{Introduction}

The CLOUD service supplier give user"s economical and climbable information storage services with a most cheaper price than ancient approaches. In ultimate cloud storage as information sharing becomes a daily feature offerings, still as Dropbox, iCloud and Google Drive, it's routine for users to share information with others during a\} very cluster to influence cloud storage services.

However,the integrity of information in cloud storage for to the ineroxable hardware/ computer code bust and human errors is contingent on skepticism and scrutiny, as knowledge remain among the cloud can merely be loose or depraved correct. to undertake and try this matter even worse, thus on beware of the identity of their services and avoid losing profits to inform users relating to these data errors the cloud service suppliers velor even be reluctant for that. Therefore, allied quest or calculation against cloud information to be checked at begining of other knowledge exertion the virtue of cloud knowledge cought.

Regain the entire knowledge from the cloud is that the regular advent for identifying knowledge correctness, then by identifying the trueness of identity or hash parameters of the entire knowledge that verify data integrity. Certainly, for to with fruition rein the truthness of cloud information this typical approach is during a vary position. However, the prowess of victimization this approach on cloud knowledge is uncertain.

The scale of cloud knowledge are vast usually is that the main reason. to identify knowledge virtue can value or perhaps waste users amounts of computation and communication resources is to downloading the complete cloud information, notably once information are corrupted among the cloud. Besides, several uses of cloud knowledge do not basically need users to pass the complete cloud data to native devices. Like Amazon, it's as a results of cloud suppliers, can provide users computation services directly on large-scale information that early presented at intervals the cloud. Recently, many mechanisms ar planned to allow not exclusively knowledge owner itself but put together a crowed patron to expeditiously do virtue checking whereas not downloading the entire knowledge from the cloud, that's remarked as public auditing. In these mechanisms, knowledge is expand within more tiny spaces, wherever entire area is severally in charge by the boss; rather than the entire knowledge is pursuived throughout virtue identifying an a stray blend of whole blocks. To utilize the owneres data via the cloud or a third-party auditor (TPA) un agency can supply virtuoso integrity checking services for this a public verifies valor even be a data user un agency would adore

During this article, we tend to propose Oruta,1 a singular privacy-preserving public auditing mechanism on shared data to resolve the on prime of privacy issue. many specifically, to construct similarity authenticators in Oruta we have a tendency to tend to utilize ring signatures, thus as that to identify the virtue of multiple knowledge whereas not retrieving the entire knowledge a public champion is prepared-while from the ultimate public champion the coherence of the signer on entire area in divide knowledge is unbroken personal. in addition, to support batch auditing, which may perform several reviewing scripts we tend to any extend our mechanism.

For several reviewing scripts at the same time and increase the adequecy of verification. Meanwhile, to preserve information privacy from crowed identifiers, Oruta is compatible with random masking that has been utilized in WWRL. Moreover, to support dynamic data we tend to together leverage index hash tables from a previous public 


\section{International Journal of Science and Research (IJSR) \\ ISSN (Online): 2319-7064}

Index Copernicus Value (2013): 6.14 | Impact Factor (2014): 5.611

auditing resolution.

\section{Literature Review}

Literature survey is the most important step in software development process. Before developing the tool it is necessary to determine the time factor, economy n company strength. Once these things $r$ satisfied, ten next steps is to determine which operating system and language can be used for developing the tool. Once the programmers start building the tool the programmers need lot of external support. This support can be obtained from senior programmers, from book or from websites. Before building the system the above consideration $r$ taken into account for developing the proposed system.

B. Wang, B. Li, and H. Li [1], with cloud information services, to be not only maintain in the cloud, however additionally shared across multiple users it's the commonplace for information. Due to the present of hardware/software faults and human mistakes the virtue of cloud knowledge is honour to skepticism. To expeditiously audit cloud information integrity while not retrieving the entire knowledge from the cloud server for this many mechanisms are develope to allow each information owners and public verifiers. However, with these existing mechanisms can inevitably affirm secreate informationidentity privacy-to public verifiers, within the crowed reviewing on the virtue of divided knowledge. During this paper, for to support crowed reviewing on divided knowledge maintain into the cloud they propose a unique privacy-preserving mechanism. Specifically, to review the truthness of divided knowledge they explode ring signatures to calculate verification data is required. With this mechanism, for information that is kept personal from crowed identifieres the coherence of the signer on entire area in shared, who are responcible for while not retrieving the whole file expeditiously checke divided knowledge virtue. additionally, rather than confirming them one by one this mechanism is ready to perform divided reviewin scripts at that time. once auditing divided knowledge virtue these experimental results demonstrate the potency and efficacy of this mechanism.

K. Ren, C. Wang, and Q. Wang [2], divert in knowledge technique cloud computing views nowadays are most energiging computing paradigm. However, to its wide adoption, protection and solitude are perceived as starting obstacles. Here, for a trustworthy public cloud environment the author"s define many essential security challenges and inspire more investigation of security solutions.

D. Song, E. Shi, I. Fischer, and U. Shankar [3], to cloud users whereas enabling wealthy applications could be a difficult task providing robust information protection. a replacement cloud dependent architecture known as information secure as a responce that is explore by Researchers,for to supply information protection that dramatically reduces the perapplication development effort, and additionally speedy development and maintenance is permitting.
C. Wang, Q. Wang, K. Ren, and W. Lou [4], the long unreal vision of computing as a utility is Cloud Computing, to relish the on-demand prime quality applications and services from a shared pool of maintainable calculating gadgets it's the place wherever users will litteraly maintain their information into the cloud. By information outsourcing, from the hedach of native information preserve and maintenance purchesers may be alleviated. However, in Cloud Computing a really difficult and probably formidable task is that the proven fact that users not have physical possession of the probably massive size of outsourced information makes the knowledge virtue protection, with departed calculating gadgets and capabilities is very for users. Thus, to an outer review group to visualize the virtue of outsourced information once required is pemitting crowed review plan for cloud information storage security is of crucial priority in order that users will resort.

B. Wang, M. Li, S.S. Chow, and H. Li [5], for to utilize the facility of cloud to do calculation on information contributed by multiple users, the hitide of cloud computing brings users verdant opportunities. because of solitude matters these cloud knowledge should be coded within several keys. However, for to single key or still faraway from sensible existing secure computation techniques are restricted. during this paper, over cloud information coded under several keys we tend to design 2 economical policies for protected exported computation.

R. Rivest, A. Shamir, and L. Adelman [6], with the novel property that in public revealing an coded key doesn't thereby reveal the corresponding uncoded key an encryption technique is given. This has 2 vital consequences: Couriers or different secure means that aren't required to transmit keys, since by the supposed recipient a message may be enciphered noting an encryption key in public discovered. since only they recognize the corresponding encoded key therefore only they will decipher the message. employing a in private control decryption key, a message may be "signed".

G. Ateniese, R. Burns, R. Curtmola, J. Herring, L. Kissner, Z. Peterson, and D. Song [7], to verify that the server possesses the initial information while not retrieving it this paper introduce a model for obvious information possession (PDP) that permits a client that has keep information at an untrusted server. By sampling dynamic bunches of areas from the server, the design outcomes probabilistic notes of possession that drastically minimises I/O prices. To check the truth the client maintains a continuing quantity of data. who decrese network interfier the challenge/response protocol pass over a little, constant quantity of information. Thus, in entierly-divided storage technique the PDP desigh for remote information checking supports massive information sets.

\section{Problem Definition and Scope}

\subsection{Problem Definition}

In our design, privacy is accomplished by permitting the parties thus it provides additional protection to permit their information in multi cloud and knowledge is divide into several components. 


\section{International Journal of Science and Research (IJSR) \\ ISSN (Online): 2319-7064}

Index Copernicus Value (2013): 6.14 | Impact Factor (2014): 5.611

\subsection{Purpose}

Cloud services provide the way of centralizing more sensitive information in to cloud. The fact that data owner \& cloud server no longer in same trusted domain may put the outsourced unencrypted data at risk \& the cloud server may leak data information to unauthorized entities, so there is need of privacy-preserving \& effective search service over cloud data also checking of integrity of outsourced data. To provide secure way for outsourcing the data in encrypted form instead of plain text. Prevent cloud server from learning additional information .Also provide multi-keyword search for getting relevance result instead of non- relevant data retrieval. To assure about accuracy and correctness of data integrity checking is provided.

\subsection{Scope}

At this time, practised and delicate software engineers acknowledge ambiguous, incomplete, or maybe contradictory necessities. For to scale back the danger that the necessities are incorrect often demonstrates live code might facilitate. an analysis of scope of the event ought to be determined and clearly expressed, once the overall necessities are gathered from the client. this can be referred to as as a scope document. sure practicality could also be out of scope of the project as a perform of value or as a results of unclear necessities at the beginning of development. This document is thought of a official document if the event is outwardly, in order that if there are ever disputes, To the client is processed any ambiguity of what were guarantees.

The title of the paper is centered $17.8 \mathrm{~mm}(0.67 ")$ below the top of the page in 24 point font. Right below the title (separated by single line spacing) are the names of the authors. The font size for the authors is 11pt. Author affiliations shall be in 9 pt.

\section{System Architecture and Design}

\subsection{System Model}

For cloud information storage in Figure 3 illustrates a representative network architecture is completely different network entities will be known as follows:

- User: users, who have trust the cloud for information computation, and additionally to be keep within the cloud comprises each individual customers and organizations.

- Cloud Service provider (CSP): a CSP, in building and managing distributed cloud storage servers CSP has vital resources and experience, Cloud Computing systems owns and operates live.

- Third Party Auditor (TPA): an elective TPA, experience and capabilities are present within the TPA that users might not have, behalf of the users upon request is sure to access and expose risk of cloud storage service. a user stores his information into a group of cloud servers through a CSP in cloud information storage, that are running during a concurrent, cooperated and distributed manner.
To additional tolerate faults or server crash as user"s information growsin size and importance information redundancy are often used with technique of erasurecorrecting code. Thereafter, for application functions, to access or retrieve his information the user interacts with the cloud servers via CSP. In some cases, on his information the user might have to perform block level operations. the foremost general types of information Flow Cloud Service provider Users Cloud Storage Servers Security Message Flow Security Message Flow Security Message Flow optional Third Party Auditor

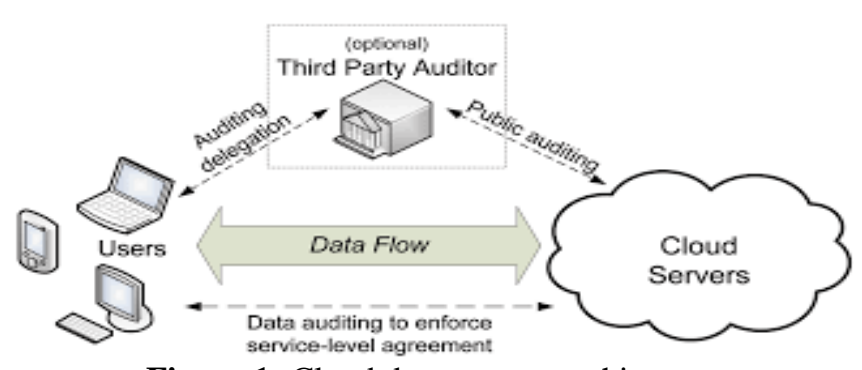

Figure 1: Cloud data storage architecture

Update, delete, insert and append we are considering these operations are block. As users now not possess their information regionally, to assure users that their information are being properly keep and maintained it's of vital importance. That is, with security suggests that so they will create continuous correctness assurance of their keep information even while not the existence of native copies users should be equipped. just in case that users don't essentially have the time, they will delegate the tasks to an optional sure TPA of their several decisions feasibility or resources their information is to observe. In our design, we assume that between every cloud server the point-to-point communication channels and also the user is attested and reliable, with very tiny overhead which may be achieved in observe. during this paper note that we have a tendency to don't address the problem of information privacy, as in Cloud Computing, to the matter we study here information privacy is orthogonal. by cloud information storage will come back from 2 completely different sources mortal Model Security threats faced. On the one hand, a CSP will be selfinterested, untrusted and probably malicious. to a lower tier of storage than in agreement for monetary reasons Not only will it want to move information that has not been or is never accessed, as a result of management errors, Byzantine failures so on however it should additionally conceive to hide an information loss incident.

\subsection{Algorithm of Proposed System}

Which functions associate exceedingly in a very given program will come back multiple leads to an economical manner In computer science within the field of compiler implementation, made product result analysis (or CPR analysis) may be a static analysis that determines. we currently assess to indicate that they're so light-weight the performance of the planned privacy-preserving public auditing schemes. For the privacy-preserving system we are going to specialise in the execution time. The experiment is 


\section{International Journal of Science and Research (IJSR) \\ ISSN (Online): 2319-7064}

Index Copernicus Value (2013): 6.14 | Impact Factor (2014): 5.611

conducted victimisation 3 formula i.e. RC5 formula, RSA formula and DES formula.

Table 1: Execution time for Files with RC5, RSA and DES Algorithm

\begin{tabular}{|l|c|c|c|c|}
\hline \multicolumn{1}{|c|}{ File name } & $\begin{array}{c}\text { File } \\
\text { Size } \\
\text { (KB) }\end{array}$ & $\begin{array}{c}\text { Executi } \\
\text { on } \\
\text { Time } \\
\text { with } \\
\text { RC5 } \\
\text { (ms) }\end{array}$ & $\begin{array}{c}\text { Executio } \\
\text { n Time } \\
\text { with } \\
\text { RSA } \\
\text { (ms) }\end{array}$ & $\begin{array}{c}\text { Executi } \\
\text { on } \\
\text { Time } \\
\text { with } \\
\text { DES } \\
\text { (ms) }\end{array}$ \\
\hline $\begin{array}{l}\text { supervisisor_1 } \\
\text { ist2012.pdf }\end{array}$ & 337 & 109.375 & 178.5 & 340.5 \\
\hline hi.doc & 10 & 125 & 200.8125 & 364.25 \\
\hline nupoor.txt & 4 & 94.3 & 120.4 & 150.54 \\
\hline $\begin{array}{l}\text { chrysanthemu } \\
\text { m.jpeg }\end{array}$ & 860 & 234.375 & 320 & 532.625 \\
\hline
\end{tabular}

For files with RC5, RSA and DES formula Table one Shows Execution Time. As compare to DES and RSA formula The execution time for RC5 is extremely less. From this we are able to say that than different 2 formula RC5 formula is economical. the subsequent Figure four shows graph illustration of on top of Table. With relevance Execution time It will show the comparison of RC5, RSA and DES formula.

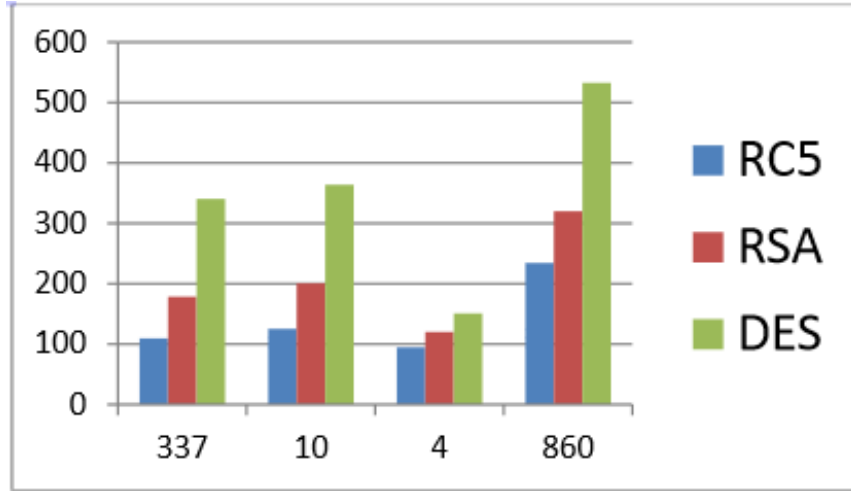

Figure 2: Comparison of RC5, RSA and DES Algorithm w.r.t Execution time

For conserving the confidentiality of the information This theme provides a watching system. Confidentiality is preserve through Third Party Auditor (TPA). conjointly it will support knowledge integrity \& validation through challenge and challenge verification.

\section{Mathematical Model for proposed work}

Input Data: file, User name, Pass

Output Data: Secured File

Process:

Encryption and upload the data to Cloud

server and query from user. Cthe

encrypted document collection stored in

the cloud server, denoted as

$\mathrm{C}=\mathrm{fc} 1, \mathrm{c} 2, \mathrm{c} 3, \mathrm{c} 4 . . \mathrm{cmg}$

Data owner encrypts the data by AES algorithm and transfer to the cloud server
Generate the random set

KyeGen For user $\mu \mathrm{i}$, he/she randomly piks $x_{i} \in \mathbb{Z}_{p}$ and computers $\mathcal{W}=g_{2}^{x i}$. User $\mu_{i}$, S public key is $p k_{i}=$

$\mathcal{W} i$ and his/her private key is $s k_{i}=x_{i}$. The original user also randomly generates a public aggregate key pak = $\left(\mathrm{r}_{1} \ldots \ldots \mathrm{t}_{k}\right)$ where, $\mathrm{n}_{1}$ are random elements of $\mathbb{G}_{1}$.

SignGen . Given all the $d$ group member , public keys $\left(p k_{1,,, y,} p k_{d}\right)=\left(w_{1},,,,,, w_{d}\right), \quad$ a block $m_{j}=$ $\left(m_{j, 1, \ldots, m_{j}} m_{j}\right)$, it's identifier $i d_{j}$, a private key $s k_{s}$ for some s, user $u_{s}$ computes a ring signature of this block as follows :

1) Aggregates block $m_{j}$ with the public aggregate key pak, and computers.

$$
\beta_{3}=H_{1}\left(i d_{j}\right) \prod_{l=1}^{k} \quad n^{j, 1} \in \mathbb{G}_{1} .
$$

2) Randomly chooses $u_{j i} \in \mathbb{Z}_{p}$ and sets $\sigma_{j, i}=g_{1}{ }^{a}, i$, for all $i \neq s$ Then Calculates,

$$
\sigma_{j, s}=\left(\frac{\beta_{j}}{\psi\left(\Pi_{i \neq s} w_{i}^{a_{j, i}}\right.}\right)^{1 / x_{s} \in \mathbb{G}_{1}}
$$

The ring signature of block $m_{j}$ is $\left(\sigma_{j, 1 \ldots, \sigma_{j, d}}\right)$.

2. Compute

$u_{i}=\sum_{i, \epsilon \mathcal{J}} y_{i} m_{i, l}+n h\left(\lambda_{l}\right) \in \mathbb{Z}_{p 1} \mu=\sum_{i} v_{i m_{i}} ;$ For $\mathrm{i} \in[1, k]$

3. Computer using.

4. Verify

$$
\left\{\mathrm{H}\left(m_{i}\right), \Omega_{i}\right\} i \in \mathrm{I}
$$

$$
\begin{aligned}
& \operatorname{sig}_{s k}(H(R)) \\
& \text { Output FALSE if fails }
\end{aligned}
$$

Proof Verify: After receiving all the B auditing proofs, the public verifier checks the correctness of these B proofs simultaneously by checking the following equation with all the $\sum_{b=1}^{B} d_{b}$ users public keys:

\section{Conclusion}

In this paper, for divided knowledge to propose a privacy protection, public among the cloud, auditing mechanisms tendency acquired by user. We tend to create authenticators homomorphic ring use signatures so a protagonist is ready to share knowledge audit All knowledge integrity, nevertheless it cannot while not retrieving the excellence is that every signer on the block. To enhance corroborative the potency of multiple auditing tasks, we have a tendency to Batch audit support to expand our network. 


\section{Future Enhancement}

As the future work, with efficiency review the virtue of divided knowledge with dynamic teams whereas still protective the coherence of the signer on every block from the third party auditor and conjointly embrace the options to alter dynamic operations (e.g. inserting/deleting knowledge block) during this system.

\section{References}

[1] B. Wang, B. Li, and H. Li, "Oruta: Privacy Preserving Public Auditing for Shared Data in the Cloud,'Proc. IEEE Fifth Int'1 Conf.Cloud Computing, pp. 295-302, 2012.

[2] K. Ren, C. Wang, and Q. Wang, "Security Challenges for the Public Cloud,'IEEE Internet Computing, vol. 16, no. 1, pp. 69-73, 2012.

[3] D. Song, E. Shi, I. Fischer, and U. Shankar, "Cloud Data Protection for the Masses,"Computer, vol. 45, no. 1, pp. 39-45, 2012.

[4] B. Wang, M. Li, S.S. Chow, and H. Li, "Computing Encrypted Cloud Data Efficiently under Multiple Keys,'Proc. IEEE Conf. Comm. and Network Security (CNS "13), pp. 90-99, 2013.

[5] The MD5 Message-Digest Algorithm (RFC1321). https://tools. ietf.org/html/rfc1321, 2014.

[6] N. Cao, S. Yu, Z. Yang, W. Lou, and Y.T. Hou, "LT Codes-BasedSecure and Reliable Cloud Storage Service,'Proc. IEEE INFOCOM, 2012.

[7] B. Wang, B. Li, and H. Li, "Certificateless Public Auditing for Data Integrity in the Cloud,'Proc. IEEE Conf. Comm. and Network Security (CNS $\left.{ }^{\text {ee }} 13\right)$, pp. 276284, 2013.

[8] C. Wang, S.S. Chow, Q. Wang, K. Ren, and W. Lou, "Privacy-Preserving Public Auditing for Secure Cloud Storage,'IEEE Trans. Computers, vol. 62, no. 2, pp. 362-375, Feb. 2013.

[9] B. Wang, B. Li, and H. Li, "Public Auditing for Shared Data with Efficient User Revocation in the Cloud,'Proc. IEEE INFOCOM, pp. 2904-2912, 2013.

[10] B. Wang, B. Li, and H. Li, "Panda: Public Auditing for Shared Data with Efficient User Revocation in the Cloud,'IEEE Trans. Services Computing, 20 Dec. 2013, DOI: 10.1109/TSC.2013.2295611.

[11]B. Wang, H. Li, and M. Li, "Privacy-Preserving Public Auditing for Shared Cloud Data Supporting Group Dynamics,"Proc. IEEE Int'1 Conf. Comm. (ICC 13 ), pp. 539-543, 2013.

[12] B. Wang, S.S. Chow, M. Li, and H. Li, "Storing Shared Data on the Cloud via Security-Mediator,"Proc. IEEE 33rd Int'1 Conf. Distributed Computing Systems (ICDCS $\left.{ }^{e} 13\right)$, pp. 124-133, 2013. 